\title{
Malignant transformation of hepatic adenomas
}

\author{
Shien TL Micchelli ${ }^{1}$, Perumal Vivekanandan ${ }^{1}$, John K Boitnott ${ }^{1}$, Timothy M Pawlik ${ }^{2}$, \\ Michael A Choti ${ }^{2}$ and Michael Torbenson ${ }^{1}$ \\ ${ }^{1}$ Department of Pathology, The Johns Hopkins University School of Medicine, Baltimore, MD, USA and \\ ${ }^{2}$ Department of Surgery, The Johns Hopkins University School of Medicine, Baltimore, MD, USA
}

\begin{abstract}
Hepatic adenomas are benign neoplasms of the liver that occur in several well-defined clinical settings, but principally that of excess hormone exposure. They have a small but poorly characterized risk of malignant degeneration. The clinical presentation and pathological findings were reviewed for all hepatic adenomas resected between January 1, 2003 and July 1, 2006. Immunohistochemistry for p53, $\beta$-catenin and $\alpha$-fetoprotein (AFP) were performed on those cases with malignant transformation and exon 3 of $\beta$-catenin was amplified and sequenced. A total of 17 hepatic adenomas were resected and 3 showed malignant transformation. All three cases were in women with an age range of 23-33 years. The clinical presentations were vague abdominal pain. Histologically, the malignant transformation occurred within otherwise typical hepatic adenomas. Two of three cases showed patchy atypia throughout the hepatic adenoma. The hepatocellular carcinoma arose as distinct nodules directly within the adenomas, effectively ruling out synchronous lesions. The hepatocellular carcinomas were unifocal in two cases and multifocal in one case with the greatest dimensions of the hepatocellular carcinoma being $2.5,2.2$, and $1 \mathrm{~cm}$. Immunostains for AFP and $\beta$-catenin were negative in both the hepatic adenomas and areas of hepatocellular carcinoma. p53 immunostaining was positive within the areas of malignant transformation in one case. No mutations or deletions were seen in exon 3 of the $\beta$-catenin gene for either the adenomas or the carcinoma. In conclusion, two of the cases that developed hepatocellular carcinomas showed cytological atypia in the background adenoma. The hepatocellular carcinomas arose as distinct nodules within the adenomas. No common molecular pathway of hepatocellular carcinogenesis was observed by examining AFP, $\beta$-catenin, and p53 immunostains and no $\beta$-catenin mutations or deletions were found.
\end{abstract}

Modern Pathology (2008) 21, 491-497; doi:10.1038/modpathol.2008.8; published online 1 February 2008

Keywords: hepatic adenoma; hepatocellular carcinoma; $\beta$-catenin

Hepatic adenomas are benign hepatocellular neoplasms classically found in livers with no evidence for underlying liver disease. The vast majority of hepatic adenomas have been reported in one of three clinical settings: (1) most commonly in reproductive age women with a history of oral contraceptive pill therapy, (2) rarely in individuals with a history of excess androgen exposure, such as androgen steroid therapy for Fanconi's anemia, and (3) also occasionally in glycogen storage disease types I and III. Rare cases of hepatic adenomas have also been reported in a number of other diverse clinical settings. The histological features and classification of hepatic adenomas have recently been expertly reviewed. ${ }^{1}$

Correspondence: Dr M Torbenson, MD, Department of Pathology, The Johns Hopkins University School of Medicine, 1503 East Jefferson, Room B314, Baltimore, MD 21231, USA.

E-mail: mtorben@jhmi.edu

Received 12 March 2007; revised 16 June 2007; accepted 18 June 2007; published online 1 February 2008
The association of hepatic adenomas with different risk factors suggests that they may be a heterogeneous group at the molecular level. To date, mutations in the TCF1 gene (also known as $H N F 1-\alpha)^{2}$ as well as dysregulation of the $\beta$-catenin pathway ${ }^{3,4}$ have been the best characterized molecular lesions in these tumors, and, in fact, appear to be mutually exclusive in most cases. For hepatic adenomas with TCF1 mutations, the adenomas are more likely to show significant fatty change and are less likely to be associated with cytological atypia or malignant changes. ${ }^{5}$ In contrast, those with $\beta$-catenin (CTNNB1) mutations are less likely to show fatty change, are more likely to show a pseudoglandular growth pattern and are more likely to show cytological atypia as well as hepatocellular carcinoma. ${ }^{5}$

In this study, we sought to fully characterize hepatic adenomas that underwent clear malignant degeneration (1) to document additional cases of this rarely reported event and (2) to investigate the possibility that the background hepatic adenoma in those cases that develop malignant degeneration 
may contain the equivalent of 'high grade dysplasia,' that is, histological features that may be useful in predicting the development of carcinoma. In this regard, others have reported areas of dysplasia in hepatic adenomas based on fine needle aspirates, raising the possibility of identifying histological precursors to cancer adjacent to the actual cancer foci in these hepatic adenomas. ${ }^{6,7}$ Finally, we sought to further examine the relationship between hepatic adenomas that develop malignancy and the presence of $\beta$-catenin mutations or deletions. To accomplish these goals, we focused on those hepatic adenomas that had histologically unequivocal nodules of hepatocellular carcinoma. A proportion of hepatic adenomas also show atypical cytological or architectural features, but because there are no follow-up data on such lesions to ascertain their natural history, we instead focused on those cases with clear malignant foci.

\section{Materials and methods}

All hepatic adenomas that underwent complete surgical resection at Johns Hopkins University Hospital between January 1, 2003 and July 1, 2006 were included in the study. Only completely resected tumors were included in this study and all needle and wedge biopsies were excluded. The clinical presentation, radiological findings, and pathology were reviewed.

Essentially nothing is known about the molecular events involved in malignant degeneration of hepatic adenomas, so additional immunostains and molecular studies of $\beta$-catenin were performed to further characterize this rare group of hepatocellular carcinomas. Immunohistochemistry for $\alpha$-fetoprotein (AFP), p53, and $\beta$-catenin were performed on both the hepatic adenomas and hepatocellular carcinomas, as these immunostains are routinely available in most large hospitals and thus potentially of diagnostic value. Overall, at least one of these immunostains is typically positive in just over half the hepatocellular carcinomas in the United States. ${ }^{8}$

Following heat antigen retrieval, $5 \mu \mathrm{m}$ sections from paraffin-embedded tissues were immunostained with AFP (Dako, Carpenteria, CA, USA; polyclonal rabbit, 1:1000 dilution), p53 (Dako, Carpenteria, CA, USA; monoclonal mouse, clone DO-7, 1:1000 dilution), and $\beta$-catenin (Transduction Laboratories, Lexington, KY, USA; monoclonal mouse, clone 14, 1:1000 dilution). The Dako EnVision + Peroxidase kit was used for immunostaining. Cases were scored as positive when at least $1 \%$ of neoplastic hepatocytes were immunolabeled.

The frequency of hepatic adenomas with $\beta$ catenin mutations is estimated to be less than $10 \%$ overall, ${ }^{1}$ but there are data to suggest that hepatic adenomas with $\beta$-catenin mutations are more likely to undergo malignant transformation. ${ }^{5}$ Thus, we also micro-dissected the hepatocellular carcinoma, extracted the genomic DNA, and amplified and directly sequenced exon 3 from both the hepatic adenoma and the hepatocellular carcinoma using methods as described previously. ${ }^{4}$ Exon 3 of $\beta$-catenin is a mutational hot spot in hepatocellular carcinomas. However, large deletions can also be found in hepatic adenomas and to examine for large deletions, additional amplification of $\beta$-catenin was performed using primers to exons 2 and 4 as described previously ${ }^{5}$ in two of the three cases (cases 2 and 3 ) with available frozen tissues.

\section{Results}

A total of 17 hepatic adenomas were resected during this time period. One was classified as a telangiectic variant of hepatic adenoma and the remaining 16 as classic hepatic adenomas. Two cases showed patchy foci of borderline reticulin loss and cytological atypia and were classified as atypical hepatic adenomas. In an additional three cases, distinct nodules of malignant transformation to hepatocellular carcinoma were found and were focused on for further study.

\section{Clinical Findings}

The three individuals were all females, aged 23, 25, and 33 years. The clinical presentations for these three cases were nonspecific, with most patients presenting with vague abdominal pain. All of the adenomas arose in the clinical setting of oral contraceptive use (Table 1). The size of the adenomas ranged from 4 to $8.5 \mathrm{~cm}$ (Table 1). Pre-operative imaging studies were available for two of the cases: one lesion showed radiographic findings typical of a hepatic adenoma and another showed findings suggestive of a hepatic adenoma with a central stellate area of low density.

\section{Background Liver Tissues}

The background livers showed mild macrovesicular steatosis in one case and moderate macrovesicular steatosis along with mild portal chronic inflammation in one case. However, no significant fibrosis was seen in the background livers in any of the cases.

\section{Histological Findings in the Hepatic Adenomas}

Despite the background fatty liver in two cases, only one of the adenomas showed minimal fatty change (Figure 1a). The adenomas were composed of bland appearing hepatocytes (Figure 1b), but patchy areas of cytological atypia were found distributed throughout the adenomas in two cases (Figure 1c). The areas of atypia showed no loss of reticulin 
Table 1 Clinicopathological findings

\begin{tabular}{|c|c|c|c|c|c|c|}
\hline Case & Age (years) & $\begin{array}{l}\text { OCP use } \\
\text { (years) }\end{array}$ & Gross findings & $\begin{array}{c}\text { Hepatic } \\
\text { adenoma }(\mathrm{cm})\end{array}$ & $\begin{array}{l}\text { Hepatocellular } \\
\text { carcinoma }(\mathrm{cm})\end{array}$ & Differentiation \\
\hline 1 & $23^{\mathrm{a}}$ & 8 & $\begin{array}{l}\text { Patchy areas of hemorrhage, no distinct } \\
\text { nodule }\end{array}$ & 4 & 1 & Moderate \\
\hline 2 & 25 & 12 & $\begin{array}{l}\text { Nodule with irregular stellate borders } \\
\text { eccentrically located in hepatic } \\
\text { adenoma }\end{array}$ & 9.5 & 2.5 & Moderate \\
\hline 3 & 33 & 15 & $\begin{array}{l}\text { Distinct nodule in center of hepatic } \\
\text { adenoma }\end{array}$ & 8.5 & 2.2 & Well \\
\hline
\end{tabular}

OCP: oral contraceptive pills.

${ }^{\mathrm{a}}$ Histologically this case shows multifocal malignant degeneration.
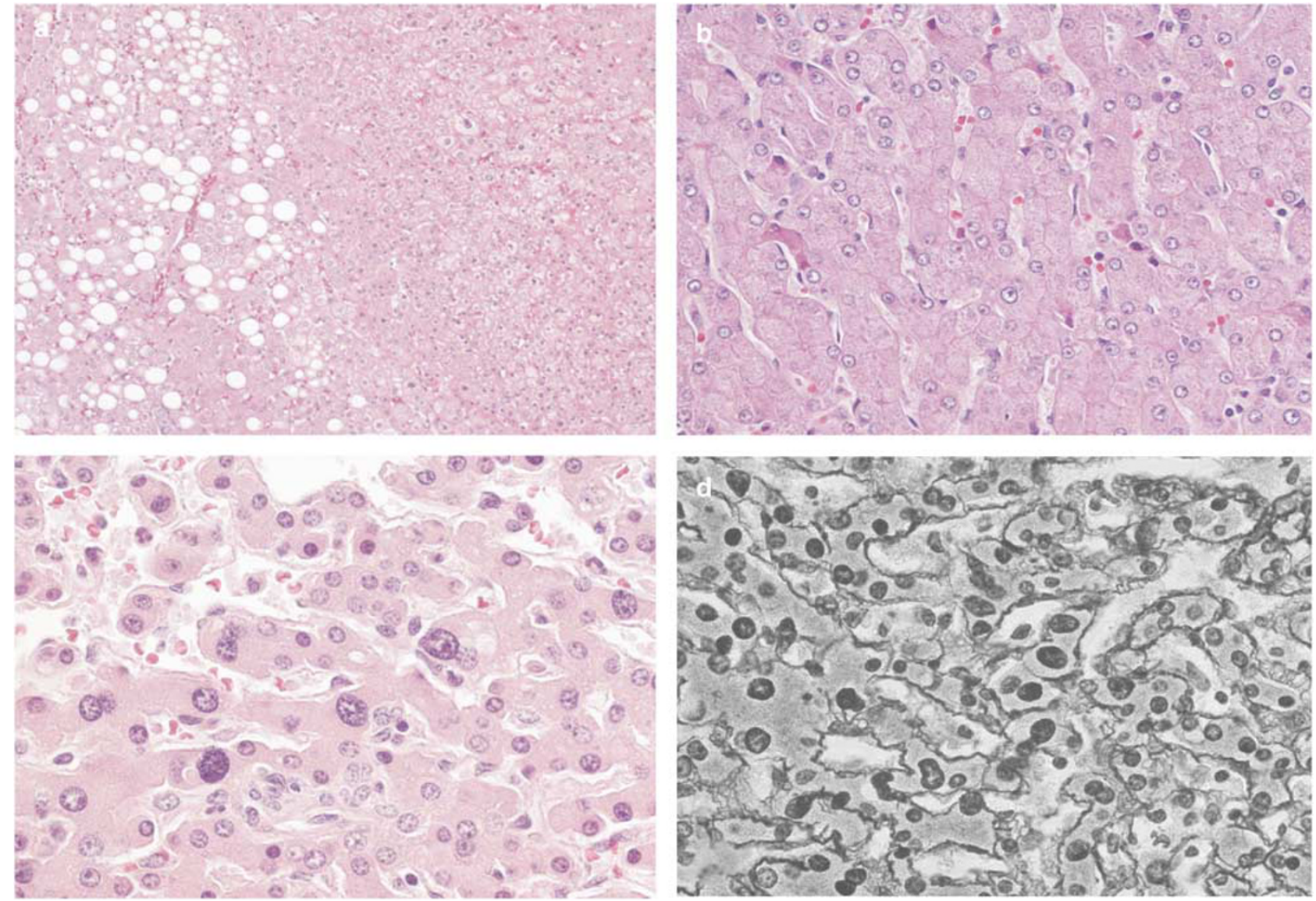

Figure 1 Histological findings in the hepatic adenomas. The hepatic adenomas showed minimal or absent fatty change. In this adenoma, shown on the right half of the image, there is no fatty change despite the presence of significant fatty change in the background liver, which can be seen on the left (panel a, original magnification $\times 40$ ); the growth pattern in all cases was trabecular (panel $\mathbf{b}$, original magnification $\times 100$ ); cytological atypia was observed in two cases, scattered in foci throughout the hepatic adenomas (panel c, original magnification $\times 160$ ); no reticulin loss was observed in the areas of atypia (panel d, original magnification $\times 160$ ).

(Figure 1d). The third case showed no atypia in the background hepatic adenoma. The cytological atypia in the adenomas with carcinoma was not clearly different from the cytological atypia seen in the two hepatic adenomas without carcinoma that were classified as atypical.
The growth pattern of the hepatic adenomas was predominately trabecular in all cases (Figure 1b). Only rare foci of pseudogland formation could be found with extensive searching in one of the cases. Other findings included areas of hemorrhage $(N=3)$, mild peliotic changes $(N=2)$ and minimal 
macrovesicular steatosis $(N=1)$, and mild patchy chronic inflammation $(N=1)$.

\section{Gross and Histological Findings of the Hepatocellular Carcinomas}

Grossly, two of the adenomas had discrete areas that corresponded histologically to malignant foci (Figure 2a). Malignant areas were unifocal in two cases and multifocal in one case (Table 1). The greatest dimension of the carcinomatous nodules ranged from 1 to $2.5 \mathrm{~cm}$. The histological grades of two of the hepatocellular carcinomas were moderately differentiated, while one was well differentiated (Figure 2b,c). Angiolymphatic invasion was not identified in any of the cases. As is typical of hepatocellular carcinoma, extensive reticulum loss was seen in all of the carcinomas (Figure 2d), in contrast to the background adenomas that retained normal reticulin patterns. In one case, the carcinoma extended focally to the surgical resection margin; re-excision of the tumor bed showed no residual adenoma or carcinoma.
Immunostains for AFP and $\beta$-catenin were negative in both the adenomas and hepatocellular carcinomas in all cases, whereas p53 immunostaining was positive within the areas of cancer in one case with approximately $25 \%$ of the nuclei showing positivity. The background adenoma was negative for p53 staining in all cases. No $\beta$-catenin mutations were detected in exon 3 by direct sequencing of either the hepatocellular carcinomas or the hepatic adenomas. In the two adenomas with frozen tissues, no deletions in the $\beta$-catenin gene were observed.

\section{Discussion}

When hepatic adenomas were first linked to oral contraceptive use in the 1970s, a discussion was initiated in regards to their malignant potential. Early investigations did not identify a significant malignant potential for adenomas and the contribution of hepatic adenomas to the prevalence of hepatocellular carcinoma was felt to be minimal or non-existent. Over the next several decades, case
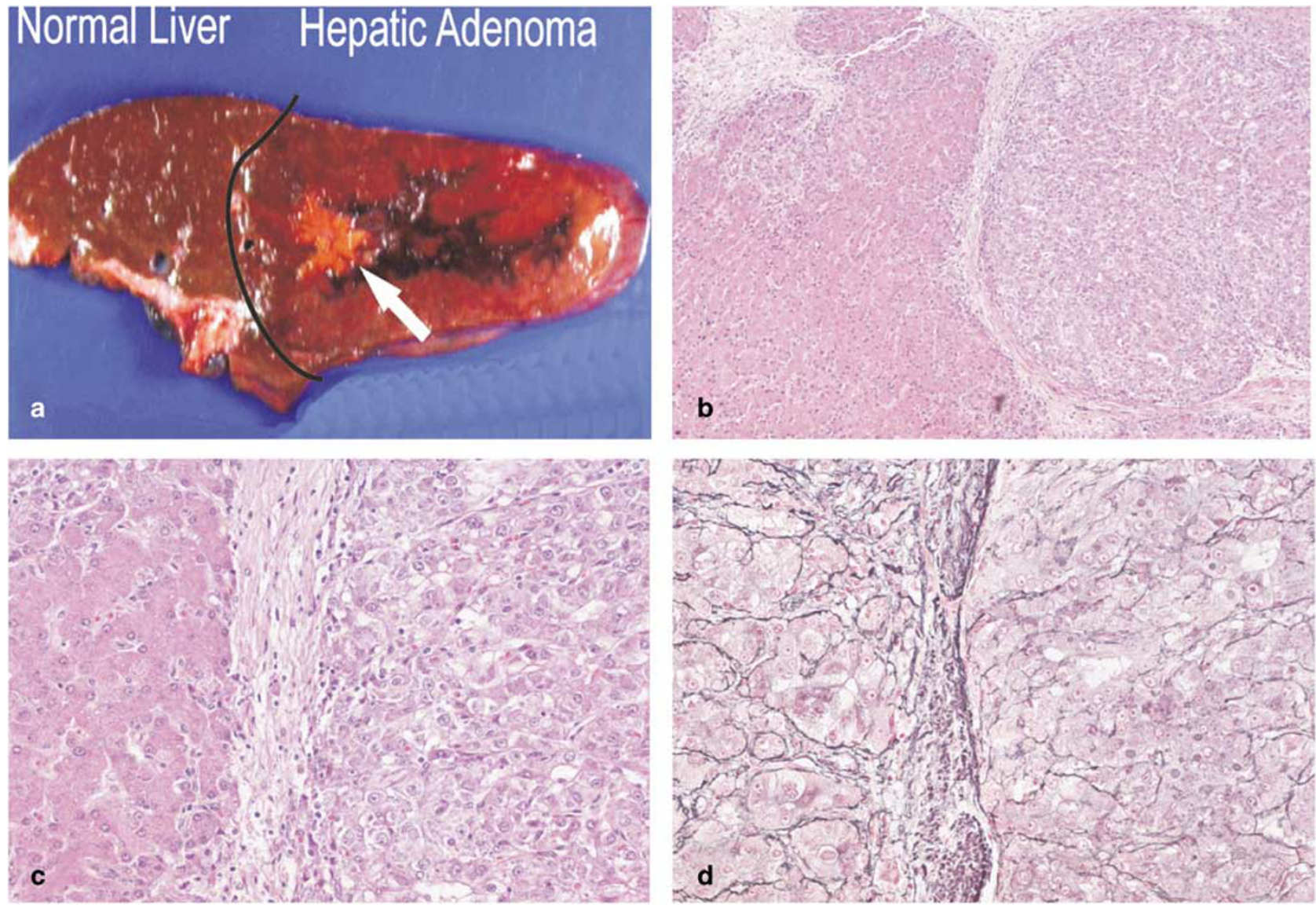

Figure 2 The hepatocellular carcinomas were recognized grossly in two cases. In this case, the carcinoma had a yellow stellate appearance as pointed out by the white arrow (panel $\mathbf{a}$, tumor size is $2.5 \mathrm{~cm}$ ); low power magnification of the interface between hepatic adenoma on the left and hepatocellular carcinoma on the right (panel $\mathbf{b}$, original magnification $\times 20$ ); the same field as panel $\mathbf{b}$ is shown at higher magnification (panel $\mathbf{c}$, original magnification $\times 64$ ); the same fields as panels $\mathbf{b}$ and $\mathbf{c}$ showing loss of reticulin in the carcinoma on the right of this image (panel $\mathbf{d}$, original magnification $\times 64$ ). 
reports were published that supported a risk for malignancy in at lease some cases (Table 2), and a small but incompletely defined risk of malignant degeneration has been generally accepted, although questions have persisted as to whether the reported carcinomas are synchronous lesions or truly arose from the within the hepatic adenoma. ${ }^{19}$ The findings in this study as well as several others in the literature with sufficient description show that hepatocellular carcinoma can at times be found directly within the adenomas. Although detailed molecular studies would be required to definitively prove a direct progression from adenoma to carcinoma, we believe that the presence of carcinoma located within the adenomatous tissue strongly argues it arose from the adenoma.

Given this malignant potential, the question naturally arises as to what proportion of hepatic adenomas develop malignancy. In this series, 3/17 $(18 \%)$ of hepatic adenomas showed the presence of carcinoma. By comparison, Foster and Berman ${ }^{17}$ reported a $13 \%$ frequency of malignancy in 39 adenomas, whereas Closset et $a l^{16}$ reported a frequency of $1 / 16(6 \%)$ of adenomas with co-existing cancer and 2/16 (12\%) with dysplasia. ZucmanRossi, in a multicenter study, found 5/93 (5\%) of hepatic adenomas with malignant degeneration and $6 / 93(6 \%)$ cases borderline for hepatocellular carcinoma. There is little additional data on this point, and none of these case series, including the current study, are of the proper design to determine the incidence of malignant degeneration in hepatic adenomas; they all have a risk for significant selection biases that may lead to an overestimation of the true frequency of malignant degeneration. Nevertheless, the frequency of malignant degeneration in the subset of hepatic adenomas that undergo resection can be estimated from these data. Based on the combined data reported in case series, 9\% of 165 resected hepatic adenomas had foci of carcinoma, with another $6 \%$ classified as 'borderline,' 'dysplastic,' or 'atypical.' As suggested in an editorial, ${ }^{19}$ a large registry of hepatic adenomas from multiple centers is likely the only way to acquire proper data to reliably define the true risk of malignancy in these tumors.

A practical question when faced with a needle biopsy of a hepatic adenoma is whether there are any histological or immunohistochemical findings in a typical adenoma that may be useful in predicting the development of carcinoma. We found cytological atypia without reticulin loss scattered in small foci throughout the background adenomas in two of three cases, suggesting that atypia may be associated with the development of carcinoma. However, despite the intuitive attractiveness of cytological atypia, data are still limited on this point and requires additional confirmation. We also examined three stains commonly available in many hospital centers and found that none of the background adenomas were positive for any of these stains. Even in the case where the hepatocellular carcinoma showed p53 positivity, there was no evidence for staining in the noncancerous hepatic adenoma tissue. Other stains, not investigated in this study, may potentially be of value. For example, glypican-3 is typically negative in hepatic adenomas but positive in hepatocellular carcinomas. ${ }^{20}$

Table 2 Reported cases of malignant degeneration in hepatic adenomas

\begin{tabular}{|c|c|c|c|c|}
\hline Report & Age (years) & $\begin{array}{l}\text { Exogenous estrogen } \\
\text { exposure (years) }\end{array}$ & $\begin{array}{c}\text { Size of } \\
\text { adenoma }(\mathrm{cm})^{\mathrm{a}}\end{array}$ & Comment \\
\hline Perret et $a l^{9}$ & 24 & 3 & 14 & \\
\hline Ferrell $^{10}$ & 29 & 6 & 18 & Discrete $5 \mathrm{~cm}$ mass arising in hepatic adenoma \\
\hline Tesluk et $a l^{11}$ & 34 & 5 & 16 & $\begin{array}{l}\text { Hepatic adenoma initially shrank to } 5 \mathrm{~cm} \text { after } \\
\text { discontinuation of OCP; tumor was resected when it } \\
\text { began to grow }\end{array}$ \\
\hline \multirow[t]{2}{*}{ Herman et $a l^{12}$} & 30 & 15 & NA & \\
\hline & 37 & 20 & & \\
\hline Gordon et $a l^{13}$ & 36 & 14 & 13 & $\begin{array}{l}\text { Hepatic adenoma apparently disappeared after } \\
\text { discontinuation of OCP; carcinoma developed in } \\
\text { same area } 7 \text { years later }\end{array}$ \\
\hline Korula et $a l^{14}$ & 40 & 15 & 6.5 & $\begin{array}{l}\text { Initial biopsy for diagnosis showed hepatic } \\
\text { adenoma; resection soon thereafter showed } \\
\text { hepatocellular carcinoma within hepatic adenoma }\end{array}$ \\
\hline Gyorffy et $a l^{15}$ & 53 & 19 & 12 & Associated with increased AFP levels \\
\hline Closset et $a l^{16}$ & 33 & 15 & 15 & \\
\hline Foster et $a l^{17}$ & 56 & 10 & 8 & $\begin{array}{l}\text { Cholangiocarcinoma in the liver; hilar lymph node } \\
\text { with cholangiocarcinoma and hepatocellular } \\
\text { carcinoma }\end{array}$ \\
\hline Ito et $a l^{18}$ & 57 & 1 month & 10 & $\begin{array}{l}\text { Abnormal imaging studies suggested development } \\
\text { of carcinoma }\end{array}$ \\
\hline Mean \pm s.d. & $39 \pm 11$ & $11 \pm 7$ & $13 \pm 4$ & \\
\hline
\end{tabular}

OCP: oral contraceptive pills.

${ }^{\mathrm{a}}$ Greatest dimension. 
At the gross specimen level, a cutoff of $5 \mathrm{~cm}$ has been suggested for recommending complete resection of hepatic adenomas. A size cutoff of $5 \mathrm{~cm}$ would have captured all of the cases in the literature (Table 2), but would have missed one of the cases from this study. A cutoff of $4 \mathrm{~cm}$ would have caught all of the cases. However, there are no well-designed studies that examine the utility of a specific size cutoff at this time point. Furthermore, interpretation of the available literature is complicated by the observation that smaller adenomas are less likely to be resected, so finding hepatocellular carcinoma only in tumors of larger size is not necessarily surprising, as few smaller tumors have been examined.

At the molecular level, significant advances were made in understanding the genesis of hepatic adenomas with initial reports of activation of the Wnt signaling pathway, ${ }^{3,4}$ followed by the discovery that TCF1 could also be mutated. ${ }^{2}$ Interestingly, either of these two mutations are seen in about $50 \%$ of all adenomas, yet are generally mutually exclusive. ${ }^{5}$ Those adenomas with $\beta$-catenin mutations have been reported to have large deletions in about one-half of the cases and mutations in exon 3 in the remaining half of cases. ${ }^{5}$ Morphologically, those adenomas with $\beta$-catenin mutations are less likely to show fatty changes, more likely to have a pseudoglandular growth pattern, and appear to be at greater risk for malignant degeneration. ${ }^{5}$ The three cases in this series did indeed show no significant fatty change, but pseudogland formation was not overtly prominent in the background adenoma. In addition, no evidence for $\beta$-catenin abnormalities were found in either the adenomas or the carcinomas: (1) no nuclear accumulation of $\beta$-catenin was observed, (2) direct sequencing of exon 3 revealed no mutations in the $\beta$-catenin gene, and (3) no deletions were found in the two cases examined. Because of the lack of frozen tissue, we were unable to completely exclude large deletions in one of the cases. These findings do not preclude a potential prognostic value for determining the $\beta$-catenin status in adenomas to determine the risk of malignant degeneration, but do underscore that all carcinomas in this setting are unlikely to follow the same pathway. Additional features, such as the presence or absence of fatty change, may be very helpful (and very practical) but require further validation.

There are several important questions about hepatic adenomas and their relationship to hepatocellular carcinoma that remain unresolved. As we have discussed in the preceding paragraphs, the histological findings, gross findings, and molecular findings each may have usefulness in predicting risk for malignancy, but none of these have been well characterized in carefully controlled studies. In addition, the biological behavior of carcinomas that arise in hepatic adenomas is unknown. Given that most have been reported in younger individuals without background liver disease and that most have been amenable to complete resection, the prognosis for these cases is likely to be considerably better than that of most hepatocellular carcinomas.

In conclusion, hepatocellular carcinoma can develop directly from within hepatic adenomas. For those individuals who undergo resection of hepatic adenomas, the frequency of malignant degeneration appears to be approximately $9 \%$ and all reported cases have been in hepatic adenomas greater than $4 \mathrm{~cm}$. The presence of cytological atypia may be a precursor lesion in some adenomas that develop malignancy. No common molecular pathway for hepatocellular carcinogenesis was observed by examining AFP, $\beta$-catenin, and p53. The utility of genotyping adenomas for $\beta$-catenin mutations and deletions to predict the risk of malignant degeneration remain unclear.

\section{References}

1 Bioulac-Sage P, Balabaud C, Bedossa P, et al. Pathological diagnosis of liver cell adenoma and focal nodular hyperplasia: Bordeaux update. J Hepatol 2007;46:521-527.

2 Bluteau O, Jeannot E, Bioulac-Sage P, et al. Bi-allelic inactivation of TCF1 in hepatic adenomas. Nat Genet 2002;32:312-315.

3 Chen YW, Jeng YM, Yeh SH, et al. P53 gene and Wnt signaling in benign neoplasms: beta-catenin mutations in hepatic adenoma but not in focal nodular hyperplasia. Hepatology 2002;36:927-935.

4 Torbenson M, Lee JH, Choti M, et al. Hepatic adenomas: analysis of sex steroid receptor status and the Wnt signaling pathway. Mod Pathol 2002;15: 189-196.

5 Zucman-Rossi J, Jeannot E, Nhieu JT, et al. Genotypephenotype correlation in hepatocellular adenoma: new classification and relationship with HCC. Hepatology 2006;43:515-524.

6 Tao LC. Oral contraceptive-associated liver cell adenoma and hepatocellular carcinoma. Cytomorphology and mechanism of malignant transformation. Cancer 1991;68:341-347.

7 Tao LC. Are oral contraceptive-associated liver cell adenomas premalignant? Acta Cytol 1992;36:338-344.

8 Torbenson M, Kannangai R, Abraham S, et al. Concurrent evaluation of p53, beta-catenin, and alpha-fetoprotein expression in human hepatocellular carcinoma. Am J Clin Pathol 2004;122:377-382.

9 Perret AG, Mosnier JF, Porcheron J, et al. Role of oral contraceptives in the growth of a multilobular adenoma associated with a hepatocellular carcinoma in a young woman. J Hepatol 1996;25:976-979.

10 Ferrell LD. Hepatocellular carcinoma arising in a focus of multilobular adenoma. A case report. Am J Surg Pathol 1993;17:525-529.

11 Tesluk H, Lawrie J. Hepatocellular adenoma. Its transformation to carcinoma in a user of oral contraceptives. Arch Pathol Lab Med 1981;105:296-299.

12 Herman P, Machado MA, Volpe P, et al. Transformation of hepatic adenoma into hepatocellular carcinoma in patients with prolonged use of oral contraceptives. Rev Hosp Clin Fac Med Sao Paulo 1994;49:30-33. 
13 Gordon SC, Reddy KR, Livingstone AS, et al. Resolution of a contraceptive-steroid-induced hepatic adenoma with subsequent evolution into hepatocellular carcinoma. Ann Intern Med 1986;105:547-549.

14 Korula J, Yellin A, Kanel G, et al. Hepatocellular carcinoma coexisting with hepatic adenoma. Incidental discovery after long-term oral contraceptive use. West J Med 1991;155:416-418.

15 Gyorffy EJ, Bredfeldt JE, Black WC. Transformation of hepatic cell adenoma to hepatocellular carcinoma due to oral contraceptive use. Ann Intern Med 1989;110: 489-490.

16 Closset J, Veys I, Peny MO, et al. Retrospective analysis of 29 patients surgically treated for hepatocellular adenoma or focal nodular hyperplasia. Hepatogastroenterology 2000;47:1382-1384.

17 Foster JH, Berman MM. The malignant transformation of liver cell adenomas. Arch Surg 1994;129: $712-717$.

18 Ito M, Sasaki M, Wen CY, et al. Liver cell adenoma with malignant transformation: a case report. World J Gastroenterol 2003;9:2379-2381.

19 Nagorney DM. Are hepatic adenomas premalignant? HPB Surg 1996;10:59-61.

20 Yamauchi N, Watanabe A, Hishinuma M, et al. The glypican 3 oncofetal protein is a promising diagnostic marker for hepatocellular carcinoma. Mod Pathol 2005;18:1591-1598. 